\title{
Representação prosódica em Yaathe. Sobre a ocorrência de pares mínimos lexicalmente marcados
}

\section{Diogo Félix Cabral}

Mestre em Linguística pelo Programa de Pósgraduação em Letras e Linguística da Faculdade de Letras, Universidade Federal de Alagoas. Trabalho concluído em 2007, financiado com bolsa de estudos da Fapeal (Fundação de Apoio à Pesquisa do Estado de Alagoas).

Resumo: Neste trabalho, analisamos, em Yaathe - uma língua nativa brasileira pertencente a tronco linguístico Macro-Jê - o padrão prosódico de palavras que têm a mesma representação segmental, mas que são diferentes em termos de significados. Nosso objetivo era buscar evidências que pudessem assegurar ao Yaathe o status de língua tonal, isto é, uma língua que usa variações de pitch distintivamente no nível lexical. A análise de dados segue de acordo com princípios de investigação acústica (LADEFOGED, 1997). Apesar de pesquisas conduzidas no último século afirmarem que 0 Yaathe é uma língua tonal (LAPENDA, 1968, por exemplo), neste estudo isso não é evidenciado.

Palavras-chave: Yaathe; Pares mínimos; Análise acústica

\author{
JANUACELE DA COSTA
}

Doutora em Letras pelo Programa de Pósgraduação em Letras da Universidade Federal de Pernambuco (2009). Professora da Faculdade de Letras e do Programa de Pós-graduação em Letras e Linguística da Universidade Federal de Alagoas.

Abstract: The goal of this paper is to analyze the prosodic pattern of words in Yaathe - a native Brazilian language belonging to Macro-Jê phylum - that have the same segmental representation, but that are different in meaning. Our aims were to find evidences for call to Yaathe the status of a tonal language, that is, a language which uses changes of pitch to distinguish meanings on the lexical level. The data analysis follows according to acoustic investigation principles (LADEFOGED, 1997). Spite of the fact that researches carried out at the last century assure that Yaathe is a tonal language (LAPENDA, 1968), in this study, however, these results are not provided. Keywords: Yaathe; Minimal pairs; Acoustic analysis 



\section{Introdução}

Falada pelos índios da tribo Fulni-ô, cuja reserva indígena localiza-se no município de Águas Belas, a cerca de 300 quilômetros de Recife, o Yaathe é uma língua indígena pertencente ao tronco linguístico Macro-Jê. Viva e falada por aproximadamente 3.700 indivíduos do grupo, segundo dados fornecidos pela FUNASA em 2006, ela desempenha funções rituais e sociais na aldeia, sendo a única língua nativa ainda falada no Nordeste do Brasil, com exceção do estado do Maranhão. (COSTA, 1999). Apesar de um amplo estudo já desenvolvido sobre o Yaathe, o conhecimento sobre o seu sistema acentual e suas representações prosódicas não é muito claro e, por isso, ainda pouco sistematizado.

Na literatura sobre o Yaathe, há afirmações, (MELAND e MELAND, 1967; LAPENDA, 1968), de que ela seria uma língua tonal, ou seja, de que estabeleceria a distinção entre determinadas palavras do seu sistema linguístico por meio de variações na cadeia melódica que, de um ponto de vista físico, são condizentes com modificações na produção do pitch ${ }^{1}$.

Dessa forma, direcionamos a elaboração deste trabalho para um estudo acústico-experimental de pares mínimos em Yaathe. Para esses pares mínimos, a distinção não se estabelece segmentalmente, desde que a sequência segmental é a mesma. Assim, o nosso trabalho terá como objetivo determinar se e que aspectos prosódicos são responsáveis pela distinção de significado entre esses vocábulos, através da identificação dos seus correlatos fonéticos.

Fenômenos acústicos de nível suprassegmental, tais como pitch, intensidade e duração, são selecionados pelas línguas naturais para o seu plano da expressão, tanto com valor distintivo-funcional, em nível fonológico ou sintático, quanto com o papel de expressar intenções não inerentemente linguístico-estruturais, como é o caso de muitas das funções pragmáticas. Pitch,

\section{Pitch é o termo técnico utilizado na análise acústica que diz respeito à frequência com a qual as cordas vocais vibram durante a produção dos sons da fala. Sua função linguística é determinada pelo tom no caso de palavras como unidade de análise e pela entonação quando temos como objeto de estudo unidades sintáticas.}


intensidade e duração são, assim, correlatos fonéticos para acento, tom e duração, quando observados de um ponto de vista fonológico.

\section{Metodologia}

Num primeiro momento, levantamos os pares mínimos apontados pela literatura disponível sobre o assunto - (MELAND e MELAND, 1967; LAPENDA, 1968) - e selecionamos palavras que eram descritas como sendo semelhantes do ponto de vista segmental e distintas do ponto de vista prosódico. $\mathrm{O}$ nosso objetivo era analisá-las experimentalmente, de modo a estabelecer o parâmetro ou parâmetros físicos responsáveis pela distinção estabelecida. Então, esses pares foram listados e nós fomos testá-los com falantes nativos.

Dois informantes, falantes nativos da língua, foram selecionados para o teste desses prováveis pares mínimos. Selecionados os pares mínimos apontados como sendo distintos devido a variações sistemáticas de pitch, o que permitia inferir para o Yaathe o status de língua tonal, iniciamos a gravação dos dados para análise acústica, o que seria feito com a utilização de um gravador MD em ambiente silencioso e com microfones direcionais.

O primeiro problema a ser enfrentado foi que os informantes atuais não reconhecem a maior parte dos tais pares mínimos. Além de esses constituírem um conjunto muito pequeno, não foram fornecidos, da forma descrita na literatura, pelos informantes da pesquisa atual. Ou esses informantes não reconheciam as formas apresentadas ou a um significado apresentado atribuíam um significante diferente, de modo que a amostra, no final, ficou reduzida a um número bastante restrito de pares mínimo, sete, para sermos exatos. 


\section{Os pares mínimos em Yaathe e a análise dos aspectos acústicos envolvidos na distinção}

Partindo da hipótese que as palavras de que dispomos são iguais, pelo menos em termos de segmento, o estudo se baseia na análise dos aspectos acústicos que são correlatos fonéticos de fenômenos distintivos de caráter suprassegmental - intensidade, frequência e duração. Por se tratar de uma análise de caráter acústico, veremos como esses correlatos se comportam nas palavras estudadas, levando em conta o aspecto de produção, focando nossa análise nas variações que são apresentadas quando comparamos um vocábulo com outro cuja representação segmental é idêntica. Para fins de simplificação, a exposição de argumentos segue de acordo com as semelhanças apresentadas para cada possível par mínimo indicado para que possamos ver mais claramente a metodologia aplicada.

$\mathrm{Na}$ Tabela 01, a seguir, apresentamos os pares mínimos coletados e analisados.

\begin{tabular}{|c|c|c|}
\hline Tabela 01 & \multicolumn{2}{|l|}{ Pares mínimos } \\
\hline Representação & & \\
\hline Segmental & Significado 1 & Significado 2 \\
\hline$\left[e \cdot t^{h} o\right]$ & rosto & avô \\
\hline [se.a] & urina & moça \\
\hline [ta.ke] & roupa & nele \\
\hline$\left[\mathrm{ta} \cdot \mathrm{t}^{\mathrm{h}} \mathrm{a}\right]$ & culpa & bico \\
\hline$\left[\mathrm{ta} \cdot \mathrm{t} \int \mathrm{a}\right]$ & lenha & espírito \\
\hline [to.le] & com & toré \\
\hline [sa.de] & coitado & é mesmo \\
\hline
\end{tabular}

Dos sete pares de palavras encontrados, apresentaremos os resultados, separando-os em quatro grupos, de acordo com o correlato fonético que parece estabelecer a distinção: 1) duração, 2) intensidade, 3) duração e intensidade e 4) um caso em que não há uma 
marcação evidente que possa levar a conclusões de ordem prosódica, como sugerido inicialmente. Segmentamos as seções seguintes de acordo com cada aspecto indicador de mudança.

\subsection{Pares mínimos que se distinguem pelo correlato fonético duração}

A sequência de segmentos [e. $\mathrm{t}^{\mathrm{h}} \mathrm{o}$ e [sa.de] apresentam, em linhas gerais, a mesma configuração prosódica, tanto em termos de intensidade quanto em termos de frequência, para os seus dois significados. As tabelas 02 e 03, a seguir, mostram os valores de intensidade e frequência obtidos, respectivamente, para cada ápice silábico dos pares estudados.

\section{TABELA 02} Intensidade para $\left[e . t^{\mathrm{h}} \mathrm{o}\right] \mathrm{e}[\mathrm{sa} . \mathrm{de}]$ Intensidade Intensidade

\begin{tabular}{lll} 
Palavra & 1ạ sílaba & 2a sílaba \\
\hline [e.t ${ }^{\mathrm{h}} \mathrm{o}$ ] rosto & $69,15 \mathrm{~dB}$ intens. & $72,05 \mathrm{~dB}$ intens. \\
\hline [e.tho] avô & $68,20 \mathrm{~dB}$ intens. & $69,59 \mathrm{~dB}$ intens. \\
[sa.de] coitado & $76,08 \mathrm{~dB}$ intens. & $70,74 \mathrm{~dB}$ intens. \\
\hline [sa.de ] é mesmo & $78,08 \mathrm{~dB}$ intens. & $68,92 \mathrm{~dB}$ intens.
\end{tabular}

Como podemos ver na Tabela 02, enquanto para [e.t $\mathrm{t}^{\mathrm{h}} \mathrm{o}$ ] a intensidade é mais baixa na primeira sílaba e mais alta na segunda, em [sa.de], há uma inversão desses valores, pois a intensidade é mais alta na primeira sílaba e mais baixa na segunda.

$\mathrm{Na}$ Tabela 03, mostramos os resultados da análise dessas palavras em termos do parâmetro acústico frequência. 
TABELA 03

Frequência para $\left[\right.$ et $\left.^{\mathrm{h}} \mathrm{o}\right]$ e [sade]

Frequência Frequência

Palavra 1álaba 2álaba

[e. $\mathrm{t}^{\mathrm{h}} \mathrm{O}$ ] rosto $\quad 129,97 \mathrm{~Hz}$ freq. $\quad 172,98 \mathrm{~Hz}$ freq.

[e. $t^{\mathrm{h}} \mathrm{o}$ ] avô $\quad 125,72 \mathrm{~Hz}$ freq. $\quad 164,92 \mathrm{~Hz}$ freq.

[sa.de] coitado $\quad 127,98 \mathrm{~Hz}$ freq. $\quad 168,56 \mathrm{~Hz}$ freq.

[sa.de] é mesmo $\quad 174,91 \mathrm{~Hz}$ freq. $\quad 18474 \mathrm{~Hz}$ freq.

Em relação à frequência, tanto na forma [e. $\left.\mathrm{t}^{\mathrm{h}} \mathrm{o}\right]$ quanto na forma [sa.de] o pitch é mais baixo na primeira sílaba e, diferente do que encontramos para o parâmetro intensidade, significativamente mais alto na segunda sílaba, quer elas signifiquem 'rosto' ou 'avô' e 'coitado' ou 'mesmo', respectivamente.

Para os dois pares apresentados, apesar de não ser possível detectar qualquer distinção expressa pela intensidade ou pela frequência, encontramos uma variação significativa em um dos elementos indicadores de aspectos prosódicos, a duração. Nesses dois casos citados, [e.t $\mathrm{t}^{\mathrm{h}} \mathrm{o}$ ] e [sa.de], tendo em vista que a duração segmental é o único elemento que se diferencia, podemos dizer que o alongamento do segmento vocálico, por ser o único aspecto com representação distinta quando comparamos as duas palavras de mesma representação, é que determina a mudança de significados entre as palavras analisadas, formando, assim, dois pares mínimos.

A variação no tempo de produção dos segmentos vocálicos que pertencem a uma unidade silábica pode conduzir, a depender do sistema linguístico e da sua organização interna, a contrastes de significado no nível lexical. É o que se verifica na análise dos pares mínimos acima analisados. Em [e.t $\mathrm{t}^{\mathrm{h}} \mathrm{O}$ rosto, o tempo de produção do primeiro segmento vocálico [e] é de 0.115598 segundos. Para esse mesmo segmento, na palavra que significa

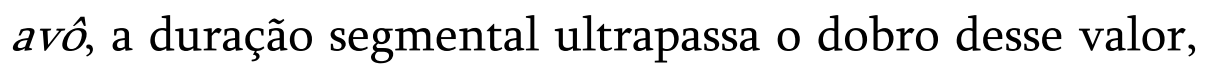
medindo 0.247657 segundos de duração. 
O mesmo fato mostrado acima é observado em relação a [sa.de]. Quando significa coitado, o segmento vocálico da primeira sílaba [a] tem um tempo de duração de 0.141896 segundos. Quando o significado é é mesmo, esse valor quase dobra, sendo de 0.269600 segundos.

Do ponto de vista auditivo, a variação na duração desses segmentos permite-nos fazer a seguinte marcação acentual, apresentada em (1) e (2), a seguir.

Deste ponto em diante, consideramos que a forma aqui analisada é a representação subjacente dessas palavras e passamos a representá-las entre barras inclinadas. Quando há uma estrutura interna reconhecida, representamos entre barras a forma dada pelo falante, com o acento, e assumimos uma representação em que há um contraste de superfície, dado que o falante demonstra conhecimento desse contraste, e damos, também, a forma subjacente, com a estrutura interna demonstrada.
(1) a. /e'tho/ avô
b. /e:'tho/ rosto

\section{(2) a. /'sade] é mesmo \\ b. /sa:'de/ coitado}

Com relação à duração, entretanto, algumas colocações são importantes, tendo-se em vista que, inicialmente, essas formas são uma representação fonética das palavras coletadas. Costa (1999) compartilha da mesma idéia apresentada por Meland e Meland (1967) de que não há, no Yaathe, segmentos longos no nível fonológico. Isso implica dizer que a distinção não seria, de fato, marcada pelo alongamento do segmento. $\mathrm{O}$ argumento utilizado é o de que as vogais longas são resultado de processos morfofonológicos que se cristalizaram na língua ao longo da sua história. Para Costa (1999):

Entretanto, no momento sincrônico, a maioria destas [vogais longas] passou a fazer parte do 
sistema fonológico da língua, desde que, embora não criem contrastes, ocorrem sem motivação evidente, quer dizer, sem que se possam predizer estas ocorrências, em um bom número de palavras da língua e, basicamente, nas mesmas posições em que são permitidas as vogais breves correspondentes. (COSTA, 1999, p.56).

Aqui, porém, parece haver uma inter-relação com a estrutura morfológica. A seguir, mostramos essa estrutura.

(3) $\left[\mathrm{e}: \mathrm{t}^{\mathrm{h}} \mathrm{o}\right]$

/e-e' $\mathrm{t}^{\mathrm{h}} \mathrm{o} /$

3Pos-avô

seu $a v \hat{o}$

(5) /'sa:de/

/sa-he-de/

EXPL-EX-ENF
(4) $\left[\mathrm{e}^{\prime} \mathrm{t}^{\mathrm{h}} \mathrm{o}\right]$

$/ \mathrm{e}^{-} \mathrm{t}^{\mathrm{h}} \mathrm{o} /$

3Pos-olho

seu olho

(6) /sa'de/

/sade/

coitado

é mesmo

Assim, mesmo a duração poderia ser contestada como tendo função distintiva em Yaathe, embora se possa considerar um contraste de superfície que pode estar sendo analisado, em alguns casos, pelos falantes como sendo realizado pelo alongamento das vogais.

O fato é que precisamos saber discernir também quando o alongamento de um segmento vocálico é resultado de processos de ordem gramatical e quando ele é produzido com função de marcar, numa sequência de unidades fonológicas, aquela cuja produção emana maior energia articulatória, definindo, assim, a unidade acentual da palavra. Apesar de termos uma distinção clara entre cada palavra marcada apenas pelo alongamento do som vocálico, tendo em vista a sua predição com base no que já foi descrito a respeito do sistema de sons do Yaathe, parece que, levando-se em conta o aspecto funcional, a duração 
desses pares analisados não seria resultado do processo de marcação prosódica. Não poderíamos, portanto, dizer que os pares de palavras constituem pares mínimos pela sua natureza acentual, mas ou por um contraste de superfície, criado por processos morfofonológicos, ou pela qualidade da vogal, admitindo-se que essas vogais são inerentemente longas. Investigações mais aprofundadas a esse respeito devem ser levadas a cabo.

\subsection{Pares mínimos que se distinguem pelo correlato fonético intensidade}

Dos pares de palavras selecionados, três apresentaram estrutura prosódica à qual poderíamos atribuir função distintiva, considerando diferenças no parâmetro intensidade. Os pares de palavras formados pelas sequências de segmentos [ta. $\left.t^{\mathrm{h}} \mathrm{a}\right],\left[\mathrm{ta} . \mathrm{t} \int \mathrm{a}\right.$ ] e [to.le], mesmo mantendo um padrão de frequência semelhante, pitch mais baixo na sílaba inicial e mais alto na final, sofrem modificações no que diz respeito ao nível de intensidade de cada sílaba analisada, como podemos ver na tabela 04 , que segue.

\begin{tabular}{|c|c|c|}
\hline TABELA 04 & \multicolumn{2}{|c|}{ Intensidade para $\left[\mathrm{ta} \cdot \mathrm{t}^{\mathrm{h}} \mathrm{a}\right],\left[/ \mathrm{ta} \cdot \mathrm{t} \int \mathrm{a}\right] \mathrm{e}[\mathrm{to} \cdot \mathrm{le}]$} \\
\hline Palavra & Intensidade 1a sílaba & Intensidade 2ª sílaba \\
\hline$\left[\mathrm{ta} \cdot \mathrm{t}^{\mathrm{h}} \mathrm{a}\right]$ culpa & $76,90 \mathrm{~dB}$ intens. & 71,91 dB intens. \\
\hline$\left[t a \cdot t^{h} a\right]$ bico & $72,65 \mathrm{~dB}$ intens. & $76,47 \mathrm{~dB}$ intens. \\
\hline [ta.t $\left.\int a\right]$ espírito & $75,71 \mathrm{~dB}$ intens. & $73,40 \mathrm{~dB}$ intens. \\
\hline [ta.tsa] lenha & 73,04 dB intens. & $77,26 \mathrm{~dB}$ intens. \\
\hline [to.le] com & $72,92 \mathrm{~dB}$ intens. & $68,68 \mathrm{~dB}$ intens. \\
\hline [to.le] toré & $67,52 \mathrm{~dB}$ intens. & $69,86 \mathrm{~dB}$ intens. \\
\hline
\end{tabular}

Os valores da tabela 04 mostram que o que diferencia uma sequência segmental da outra em termos de análise acústica é a variação de intensidade. Enquanto que para uma das representações a intensidade é mais forte na primeira sílaba, onde temos a sensação auditiva de acento, para a outra a marca 
de proeminência se dá na sílaba final. Levando em conta a não variação dos outros aspectos estudados frequência e duração - podemos atribuir à intensidade a responsabilidade por tal diferenciação de significado. As medidas de duração para os pares estudados não são relevantes, uma vez que há uma grande proximidade entre os valores. Nesse caso, precisamos levar em conta que, mesmo quando a variação existe, ela precisa ser significativa para estabelecer uma caracterização que seja perceptível em termos de análise acústica.

Para o par apresentado em (6), encontramos 76,90 dB intensidade na primeira sílaba e 71,91 dB de intensidade na segunda sílaba para (6a); 72,65 na primeira sílaba e 76,47 na segunda sílaba para (6b), o que mostra a diferença acentual entre os dois pares ${ }^{2}$. Pode-se argumentar que esses valores são aproximados, mas, de um ponto de vista fonológico, a percepção de proeminência não precisa ter correlatos físicos expressivos. Do ponto de vista auditivo, o falante nativo confirma os resultados experimentais, pois, quando testados, são capazes de identificar a sílaba acentuada, ou seja, a mais forte. Além disso, devemos levar em consideração que esses pares mínimos foram fornecidos pelos falantes espontaneamente, pois, como dissemos anteriormente, os pares de palavras que tentamos elicitar não foram reconhecidos pelos falantes.

Assim, assumimos que, considerando-se a intensidade, as palavras descritas devem ser representadas como em (6), abaixo.

(6) a. /'tat ${ }^{\mathrm{h}} \mathrm{a} /$ culpa

b. /ta' $\mathrm{t}^{\mathrm{h}} \mathrm{a} /$ bico

Para o par a seguir apresentado como (7), os resultados foram os seguintes: i) a forma (7a) foi pronunciada com $75,71 \mathrm{~dB}$ de intensidade na primeira sílaba e 73,40 dB de intensidade na segunda sílaba, o que nos mostra o acento na primeira sílaba, ou oxítona; ii) a forma (7b) teve 73,04 dB de intensidade na primeira
2 Em Cabral (2009), ficou demonstrado que as palavras em Yaathe são oxítonas ou paroxítonas, parte definida lexicalmente e parte morfologicamente. 
sílaba e 77,26 dB de intensidade na segunda sílaba e isso nos dá uma palavra com acento na segunda sílaba, ou paroxítona.

(7) a. /'tat $\int \mathrm{a} /$ espírito

b. /ta't $\int \mathrm{a} /$ lenha

A situação descrita é, basicamente, a mesma para o segundo par, apresentado a seguir, como (8). A forma (8a) foi pronunciada com 72,92 $\mathrm{dB}$ de intensidade na primeira sílaba e $68,68 \mathrm{~dB}$ de intensidade na segunda sílaba, o que nos dá uma palavra paroxítona. Para as formas em (8b) obtivemos valores relativos invertidos: $67,52 \mathrm{~dB}$ de intensidade para a primeira sílaba e 69,86 dB de intensidade para a segunda sílaba, uma palavra oxítona.

(8) a. /'tole/ com (posposição)

b. /to'le/ toré(dança dos Fulni-ô)

Desse grupo, portanto, podemos afirmar que a intensidade é que determina a sensação auditiva da realização do acento, sendo, por isso, o correlato responsável pela distinção de significados. Não encontramos pistas que nos permitam, sincronicamente, buscar explicação na estrutura interna dessas palavras.

\subsection{Pares mínimos que acumulam os correlatos fonéticos intensidade e duração}

Em um único caso, encontramos um par de palavras cuja distinção prosódica é definida conjuntamente pela duração e intensidade. Os significados conduzidos pela sequência segmental [se.a] possuem marcação acentual em posições distintas, sendo essa marcação definida por um maior prolongamento da vogal que constitui o núcleo da segunda sílaba e um maior nível de intensidade. Representamos abaixo, no exemplo (9), a marcação indicada: 
(9) a. /'se:.a] urina

b. /se'a:/ moça

Esse exemplo, único reconhecido pelos falantes como constituindo um par mínimo e que, submetido à análise experimental, mostrou os resultados acima descritos, é um dos apontados por estudos anteriores, conforme dissemos antes neste trabalho. Para demonstrar a função distintiva do tom - realizado fisicamente pelo parâmetro acústico frequência - acrescentava-se a esse par uma terceira palavra, formando-se um tríplete mínimo que, entretanto, era o único exemplo em Yaathe para essa presumida distinção tonal no nível do léxico.

O terceiro membro dessa oposição era dado como [se.a] algodão, que não foi reconhecido pelos falantes nativos que participaram da nossa pesquisa.

\subsection{Um caso de homofonia?}

Os pares de palavras selecionados foram coletados e identificados como possíveis pares mínimos, levandose em conta o conhecimento inato que os informantes possuem sobre a língua Yaathe. Na listagem apresentada, entretanto, um par de palavras, após analisado, não mostrou qualquer tipo de variação nos aspectos possíveis intensidade, frequência e duração - para que pudéssemos atribuir a diferença de significado a algum desses elementos de ordem prosódica.

Para o significante [ta.ke], as duas palavras significadas - roupa e nele - apresentaram maior intensidade e frequência na sílaba final, sendo a duração irrelevante, nesse caso, pela proximidade existente entre elas. A sensação auditiva indica também uma acentuação marcada na última sílaba para os dois casos. Com base nesses dados, portanto, poderíamos afirmar que esse par submetido à análise não forma um par mínimo ideal, uma vez que não há nenhum traço que possa marcar a diferença de significados entre as palavras. É evidente, 
porém, que, de um ponto de vista auditivo, existe uma distinção entre elas. Essa diferença, ao que parece, seria apenas de qualidade vocálica.

O último som vocálico analisado na produção de [take] nele é marcado por uma queda mais acentuada da frequência, o que seria, provavelmente, um indicativo de redução da pressão subglótica. A razão para isso, possivelmente, pode ser o fato de o advérbio nele ser a junção de dois morfemas, conforme mostramos abaixo, em (10), comparando a estrutura interna de [take] nele, com [take] roupa.

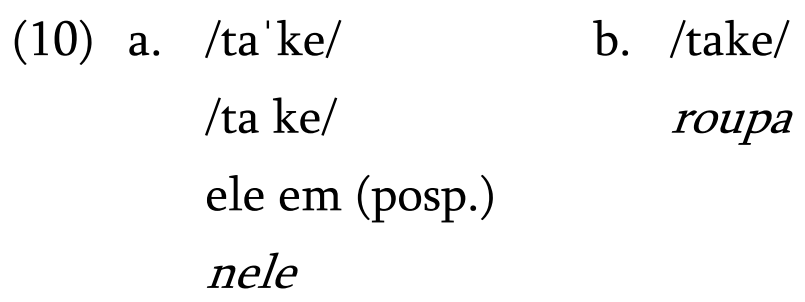

Dessa forma, quando justapostos, apenas uma dessas unidades mantém a proeminência. Não temos, portanto, um par mínimo formado.

\section{Conclusão}

A observação dos pares analisados conduz a algumas conclusões a respeito da língua Yaathe e da sua organização prosódica.

Em primeiro lugar, nos casos em que a modificação de significado é condicionada por variações de duração, a duração no tempo de produção desses segmentos não pode ser considerada um aspecto de ordem prosódica porque ela é o resultado de processos morfofonológicos. Levando em conta aspectos de descrição morfofonológica, então, o alongamento do som vocálico nos casos especificados seria resultado de processos gramaticais, não havendo ligação alguma com a marcação prosódica. Poderíamos, assim, considerar que os sons vocálicos mais longos, mesmo que resultados de processos morfofonológicos, estariam agora, no momento sincrônico da língua, exercendo função 
distintiva. Contudo, não podemos dizer isso com relação às palavras aqui analisadas, dado que a sua estrutura interna ainda é transparente.

O estudo, ainda, nos leva a outra conclusão. As afirmações sobre o Yaathe ser uma língua tonal, ou seja, uma língua que usa variações de frequência - pitch - com função distintiva entre itens lexicais, não se sustenta. Inicialmente, tivemos dificuldade para conseguir na coleta de dados as mesmas palavras que foram apresentadas como pares mínimos em outros estudos. Em todos os casos estudados, o valor da frequência era sempre ascendente baixo na primeira sílaba e alto na segunda, ou seja, um padrão bastante regular. Temos, portanto, um único elemento atuando determinadamente nesse processo de mudança de significado, a intensidade, em quase todos os casos. No último caso, em que a sílaba acentuada, além de apresentar maior intensidade, possui também maior duração no segmento acentuado, um dos membros do par possui estrutura interna, o que anula a hipótese de haver aí um par mínimo fonológico.

A intensidade, por outro lado, parece possuir status linguístico, distintivo, já que foi o único correlato identificado para a determinação da distinção de significado em alguns dos casos apresentados.

A utilização de acento com função lexical nas línguas é, como já mencionamos, um fato recorrente. O reconhecimento dos aspectos prosódicos e de sua organização, entretanto, é uma questão fugidia. O objetivo primeiro da pesquisa é identificar os traços prosódicos foneticamente para que possamos, por meio desse estudo, estabelecer que critérios são utilizados para que a intensidade, frequência e duração se agrupem em um arranjo sistemático que possa trazer informações sobre a organização acentual do Yaathe. Definir essa organização levando em conta um número reduzido de dados não é tarefa fácil. As conclusões a que podemos chegar podem estar fundadas em bases não tão sustentáveis. 
Assumiremos, por essa razão, os dados apresentados como um todo, para fim de análise, mas tendo em mente que as argumentações expostas seriam mais uma fonte de indícios para estudos posteriores, baseados em uma nova coleta, do que, necessariamente, conclusões fechadas a respeito do aspecto estudado. 


\section{Referências}

CABRAL, Diogo Félix. O acento lexical em Yaathe.

(Dissertação de Mestrado). Maceió: UFAL, 2007.

COSTA, J. F. Ya:thê, a última língua nativa no Nordeste do

Brasil: aspectos morfofonológicos e morfossintáticos. (Tese de

Doutorado). Recife: UFPE, 1999.

FOX, A. Prosodic Features and prosodic structure: the phonology of supresegmentals. New York, USA: Oxford University Press, 2002.

GUSSENHOVEN, C. The phonology of tonal and intonation. United Kingdom: Cambridge, 2005.

HAYES, B. Metrical stress theory: principles and case studies. Chicago: University of Chicago Press, 10994.

LADEFOGED, P. A course in phonetics. 4. ed. Boston, USA: Heinle \& Heinle, 2001.Chicago: The University of Chicago

LADEFOGED, P. Elements of acoustic phonetics. Chicago: The University of Chicago Press, 1996.

LAPENDA, G. Estrutura da lingua Yatê. Recife: UFPE, 1968.

MELAND, D. e MELAND, D. Fulni-ô (Yathe) phonology statement. Arquivo linguístico n. 025. Brasília, D. F.: Summer Institute of Linguistics, 1967. 
